\title{
Sensitivity to pictorial shape perspective in 5-year-old children and adults
}

\author{
RICHARD K. OLSON, MARC PEARL, NANCY MAYFIELD, and DROW MILLAR \\ University of Colorado, Boulder, Colorado 80302
}

\begin{abstract}
Five-year-old children's and adults' sensitivity to shape perspective was tested with relative length judgments of two crossed lines on the surface of a tilted "box." The stimuli were presented in both slides and three-dimensional viewing conditions. Judgments in the three-dimensional condition corresponded closely to the actual three-dimensional lengths. Responses in the slide-viewing condition were similar for both age groups, and showed about a one-third regression toward pictureplane length judgments. The lack of age effects was considered with respect to theories of the development of sensitivity to shape perspective.
\end{abstract}

Sensitivity to binocular disparity and motion parallax information for visual depth has been demonstrated in infants younger than 4 months, but the ability to use pictorial depth information apparently develops after this period (Bower, 1965; Day, 1974; Yonas, Note 1). Recent research on the development of children's depth responses to pictorial information has shown strong sensitivity to interposition and height-in-the-picture-plane in 2-year-olds (Olson \& Boswell, 1975), and a developmental trend in sensitivity to picture-plane size for children between 3 and 5 years of age (Olson, 1975). The present study focuses on the development of sensitivity to a fourth major category of pictorial depth information: shape perspective.

Shape perspective information for depth and slant is derived from the geometric regularities of our environment. For example, a "wheel" photographed at an angle to the camera's line of sight will produce an ellipse on the picture plane, and the angle from the line of sight may be derived from the amount of elliptical distortion by a very simple cosine function. There is abundant evidence that adults are able to utilize shape perspective information in judgments of slant. Olson (1974) presented trapezoidal outlines to adult subjects and found reasonably accurate judgments of the perspective implied slant when the subjects assumed that the outlines represented slanted rectangles. Attneave and Frost (1969) tested subjects' slant responses to the perspective information contained in the picture plane projections of the outlines of a box. Their subjects were also able to use per-

Portions of this paper were read at the meeting of the Psychonomic Society, Denver, Colorado, November 1975. We thank Carol Stewart for assistance in pilot work, and Vic Wesely and Dorothy Holloway for assistance in data analysis. Supported by the council on Research and Creative Work at the University of Colorado and the Institute for the Study of Intellectual Behavior. Requests for reprints should be sent to Richard Olson, Department of Psychology, University of Colorado, Boulder, Colorado 80309. spective to judge implied slant, although the slant judgments regressed somewhat toward the pictureplane from the geometrically implied slant.

The response measures in the above studies required the subjects to set a binocularly viewed rod to be collinear (Attneave \& Frost, 1969) or coplanar (Olson, 1974) with the implied three-dimensional orientations of monocularly presented two-dimensional outline figures. These methods proved to be quite suitable for adults. However, a developmental study using both the collinear and coplanar judgments failed to show systematic responses to shape perspective information in young children (Olson, Note 2). Four-year-olds were extremely variable in their slant responses and showed only a slight tendency to respond to the perspective information. Eight-yearolds' responses showed a much closer relation to the perspective-implied three-dimensional orientation, although they were substantially more variable than an adult group. We tentatively concluded from these studies that there was a developmental trend in children's sensitivity to shape perspective which began around 4 years of age. However, the 4-yearolds' failure to respond to shape perspective information may have been related to the method of judgment. The collinear and coplanar adjustments of the response rod required some degree of patience and concentration, and it often seemed that the younger children were unable or unwilling to comply with the task demands. Therefore, in the present study, we turned to a much simpler but less direct response which involved a judgment of which of two lines was longer or shorter. The two lines formed a cross which was positioned on the top surface of a slanted box as in Figure 1. The subjects' sensitivity to shape perspective was tested by presented slides of the boxes slanted at different angles from the picture-plane. As the box slant was increased, the vertical:horizontal cross-arm length ratios were decreased in the picture plane. A lack of sensitivity to shape perspective 


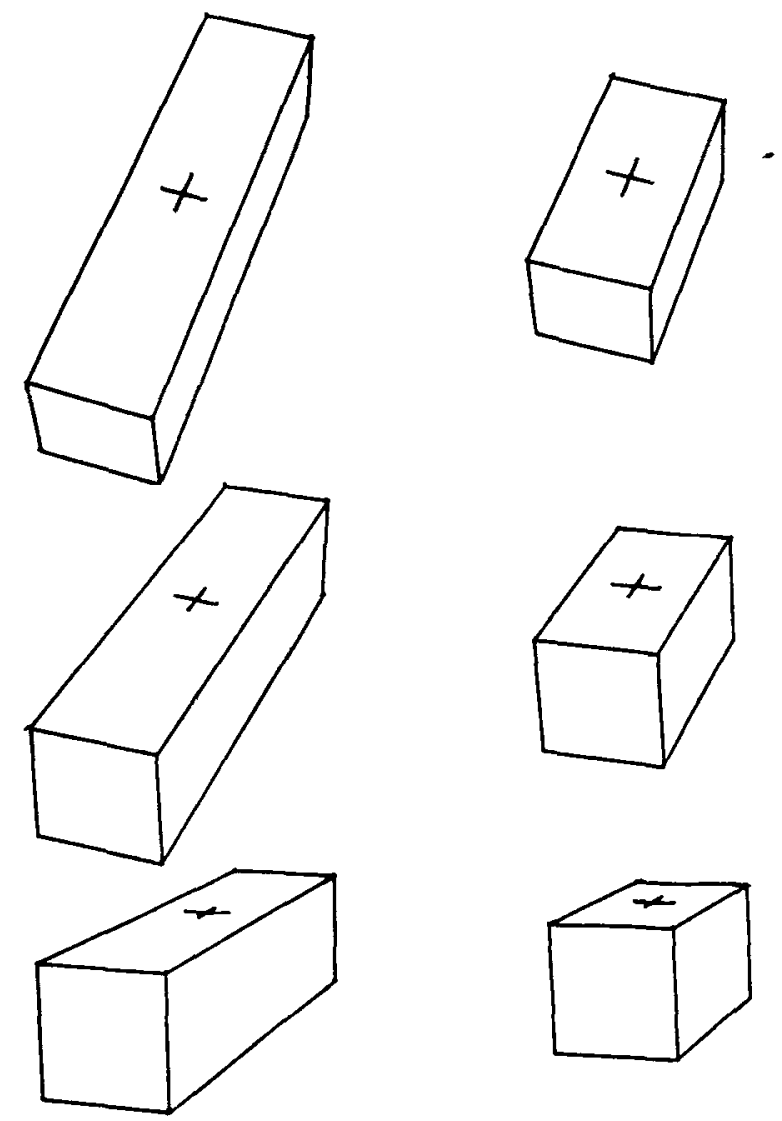

Figure 1. The box and cross stimuli. Four-to-one box shape ratios on the left and two-to-one on the right. Top boxes tilted at $40 \mathrm{deg}$, middle boxes tilted at $60 \mathrm{deg}$, and bottom boxes tilted at 80 deg from the picture-plane. Crosses are one-to-one threedimensional length ratios.

would be indicated if the subjects' judgments of the line lengths corresponded to the lengths in the picture-plane, while a perfect response to shape perspective would be indicated if the responses corresponded to the represented three-dimensional lengths.

Two additional conditions were included in the main experiment. First, the box and cross stimuli were also presented to each subject in an unrestricted three-dimensional viewing condition so that the effects of reducing the depth information to shape perspective could be compared with the threedimensional view. Second, the cross stimuli were presented on two different-sized boxes to see if the subjects' judgments were influenced by the relative distances between the cross arm ends and the box edges. Finally, additional groups of subjects were tested in two control conditions, one with the crosses in the frontal plane to check for potential response biases without perspective distortion, and a second condition with slides of the crosses at a 60 -deg angle and the box contours masked to see if the subjects' pictorial length responses were exclusively related to box shape perspective information.

\section{METHOD}

\section{Subjects}

Participants in the main experiment included 16 children (mean age $=58.8$ months, $\mathrm{SD}=7.3$ ), who were residents in the married student housing project at the University of Colorado, and 16 adults from an introductory psychology class.

\section{Stimuli and Apparatus}

Two boxes with dimensions of $125 \times 125 \times 250 \mathrm{~mm}$ and $125 \times 125 \times 500 \mathrm{~mm}$ were constructed from plywood and painted white, with 3-mm black lines along the edges. The boxes were mounted to a 25 -mm-diam black dowel which passed through the box at a 70-deg angle to the sides and just below the middle of the box top. A stand and clamp at each end of the dowel allowed settings of the box top surface at angles between 0 and 90 deg to the observer's and camera's line of sight. A pointer and protractor at one end of the dowel allowed a box angle positioning accuracy of $\pm 1 \mathrm{deg}$.

The length judgment stimuli were 2-mm-wide $\times 1$-mm-thick balsa strips, which were glued in a cross configuration, with one cross piece painted green and the other painted red, and a white dot at the cross junction. A $12-\mathrm{mm}$ piece of balsa was glued to the back of the cross junction to allow positioning of the cross in a small hole at the center of the box top (see Figure 1). Eleven crosses were constructed to the vertical:horizontal length ratios of $4: 1,3: 1,2: 1,1.75: 1,1.5: 1,1.25: 1,1: 1,1: 1.25,1: 1.5,1: 1.75$, and $1: 2$, with the constraint that both arms of the cross total $100 \mathrm{~mm}$ in length. Counterbalancing of horizontal and vertical cross-arm colors yielded a complete series of 22 cross stimuli.

The boxes were suspended above a $60-\mathrm{cm}$-high table so that the cross center was $110 \mathrm{~cm}$ above the floor. The camera lens and subject eye position were also $110 \mathrm{~cm}$ above the floor and $1.5 \mathrm{~m}$ from the cross point. Kodachrome II Professional slides were taken of each of the 22 cross stimuli on the two boxes, with the box top surfaces set at 40-, 60-, and 80-deg angles from the frontal plane (see Figure 1). The boxes were illuminated on all visible surfaces from several different light sources and photographed against a black background so that there was no consistent shading information for the box surface orientations. Also, there was no visible texture on the box surfaces.

The slide stimuli were projected on a rear-projection screen placed at the front of the table and $1.4 \mathrm{~m}$ from the observer's eye. The subject's retinal images from the slides were nearly equivalent to those obtained from the three-dimensional stimuli. Both threeand two-dimensional stimuli were viewed through a $15-\mathrm{cm}$-wide $x$ 10-cm-high window which was closed by a shutter during stimulus changes.

\section{Procedure}

The subject first viewed a series of conceptual qualifying slide stimuli containing the long box oriented in the frontal plane and vertical:horizontal cross ratios of $2: 1$ and $1: 2$. He was asked to report the color of either the longer or shorter of the two lines, and was given feedback regarding the correctness of his responses. All adults and nearly all children reached a criterion of six consecutive correct responses in the first six trials. The subject was then transferred to either the three-dimensional box or slide viewing condition and was asked to continue making the same judgment about the crosses on the tilted boxes. A total of 66 stimuli were viewed by each subject, with a 5 -min break between the slide and three-dimensional box stimulus series. The whole session usually lasted about $1 / 2 \mathrm{~h}$. All subjects' responses were rewarded with verbal praises from the experimenter, with some extra enthusiasm for the children, and the children were given a small toy at the end of the experiment.

\section{Design}

Box size was a between-subjects variable, with eight subjects from each age group in each box-size condition. Viewing con- 
dition, box angle, and cross ratios were all within-subjects variables. Orders were counterbalanced as follows. Experimental convenience dictated that the slide and three-dimensional stimuli be shown in two separate series. Thus, the order of slide and three-dimensional series was counterbalanced over subjects. Trials at each box angle were also grouped for convenience, with box angle orders of either $40-60-80 \mathrm{deg}$ or $80-60-40 \mathrm{deg}$ counterbalanced over subjects. The order of presentation for the 11 cross ratio lengths at each box angle was randomized. Also, red-vertical/ green-horizontal or green-horizontal/red-vertical crosses were randomly chosen for each trial.

\section{Control Conditions}

Two control conditions were run with different groups of subjects. The first condition included four children (mean age $=$ 57.1 months, $\mathrm{SD}=3.4$ ) and four adults from the populations described under Subjects. They viewed a randomly ordered series of 22 slides containing two presentations of each of the 11 cross ratios from the main experiment. The crosses were placed on the long box with the top surface oriented in the frontal plane. The subjects judged which of the cross arms was longer or shorter, as in the main experiment.

The second control condition included four children (mean age $=57.1$ months, $\mathrm{SD}=3.4$ ) and four adults, also from the populations described under Subjects. These subjects viewed the 11 ratios from the 60 -deg long-box condition twice and in random order. The slides were masked so that the only visible contours were the crosses. Again, the judgments were which of the lines was longer or shorter. We were interested in their spontaneous judgments from the visual information, so the subjects were not told that the stimuli were right-angle crosses pictured at various slants.

\section{RESULTS}

\section{Derivation of Apparent Equal Ratio Values}

Each subject's responses to the vertical and horizontal ratio series were summarized within each condition by adding the number of responses specifying the vertical line as longer, and these totals were entered into all of the analyses of variance described below. The graphed values in Figure 2 represent the mean interpolated ratio transition values where the subjects' mean responses would have been evenly divided between horizontal-longer and vertical-longer judgments. Interpolation was required because there were no actual cross-arm stimulus ratios corresponding to these values. The equal ratio value estimates were based on the average number of vertical-longer judgments in two adjacent cross-arm ratio conditions. For example, if the subjects responded that the vertical was longer $40 \%$ of the time for the 1.25:1 ratio, and $80 \%$ of the time for the 1.5:1 ratio, the interpolated $50 \%$ and apparent equal ratio value would be 1.31:1. These equal ratio values were graphed rather than the total vertical-line-longer judgments in the ANOVA, because they more meaningfully represent the subjects' performance. A graph based directly on the number of vertical-longer judgments was nearly identical to the pattern seen in Figure 2.

\section{Control Experiments}

The subjects' summed vertical-longer responses in the frontal plane box-orientation control condition were entered into a one-way (age) analysis of variance $[F(1,6)=.21 ; p>.05]$. Thus, the two age groups were very similar in their performance, and the estimated equal ratio values for adults (A) and children $(\mathrm{C})$, graphed for the 0 -deg condition in Figure 1, were very close to the correct $1: 1$ ratio value. The results of this condition exclude the possibility that the subjects' length judgments were significantly biased by a horizontal-vertical illusion or by the differences in distance between the crossarm ends and the box sides.

Responses in the 60-deg box-angle masked control condition were also entered into one-way (age) analysis of variance $[F(1,6)=.53 ; p>.05]$. Again, there was no significant age difference and the interpolated equal ratios based on the subjects' responses were very close to the equal picture-plane ratios (see Figure 2). This indicates that the tendency to respond to the implied three-dimensional lengths in the slide condition of the experiment was caused by the presence of the box-shape perspective information.

\section{Main Experiment}

Preliminary statistical tests and graphing of related means and interactions indicated that performance in the two box-size conditions was nearly identical in all respects, so the data were combined across box size in the following analysis. The subjects'

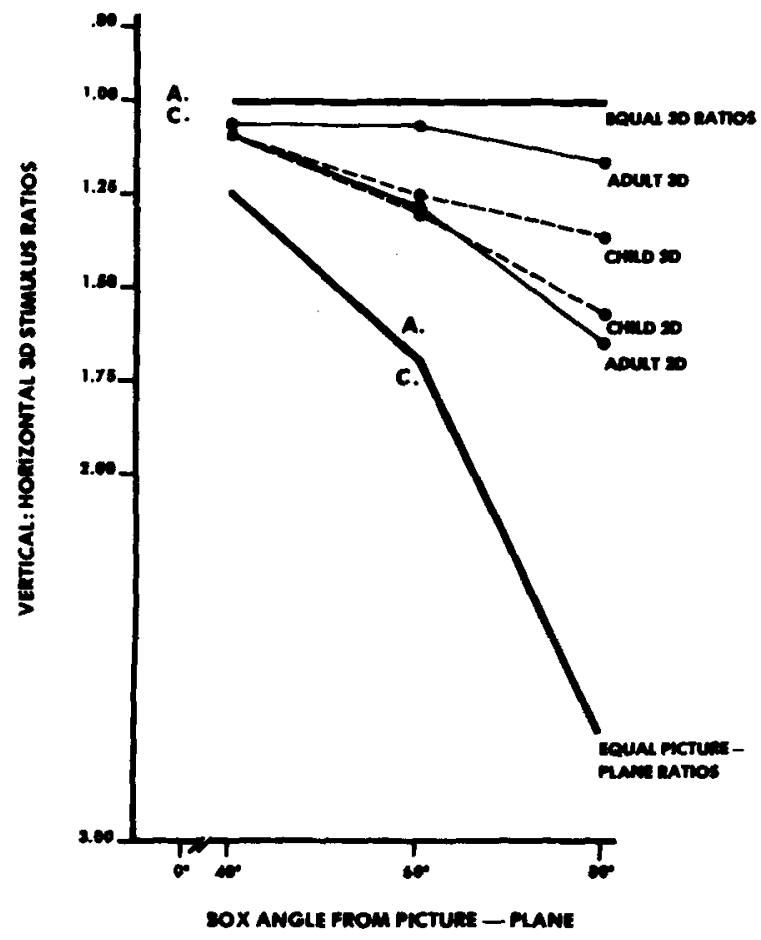

Figure 2. The subjects' mean interpolated $50 \%$ ratio transition values. $A$ and $C$ refer, respectively, to mean adult and child responses to control conditions: $3 D$ refers to the unrestricted box viewing condition; $2 \mathrm{D}$ refers to the slide viewing condition. 
summed vertical-longer responses in each category were entered into a 2 (age) by 2 (slide vs. threedimensional view) by 3 (box angle) analysis of variance. The mean apparent equal ratio values are plotted in Figure 2.

There was no significant age main effect $[F(1,30)$ $=1.75 ; \mathrm{p}>.05 \mathrm{~J}$, although age interacted with the viewing condition $[F(1,30)=7.04 ; p<.05]$, with adults showing a larger difference between viewing conditions.

The significant effects for slide vs. threedimensional viewing condition $[F(1,30)=27.32$; $\mathrm{p}<.001]$ and box angle $[\mathrm{F}(2,30)=53.31 ; \mathrm{p}<.001]$ are obvious in Figure 2 . The interaction between viewing condition and box angle $[F(2,60)=10.30$; $\mathrm{p}<.01]$ was reflected in the increased separation between slide and three-dimensional viewing conditions as the box angle increased from the frontal plane. This interaction was produced by the increased range between equal three-dimensional and equal picture-plane ratios as box angle increased, and by differences between the two viewing conditions in proportional regression toward the picture plane. The averaged apparent equal ratio regression toward the picture plane ratios in the slide viewing condition was a fairly constant proportion over the 40-, 60-, and $80-\mathrm{deg}$ box angles $(.37, .39$, and .35 , respectively), while in the three-dimensional conditions the averaged proportional regressions were $.32, .22$, and .14 , respectively. Both the Age by Box Angle $[\mathrm{F}(2,30)=.57]$ and Age by Box Angle by Viewing Condition $[F(2,60)=1.54]$ interactions were nonsignificant $(p>.05)$.

Differences from the boundary conditions of equal three-dimensional ratios and equal picture-plane ratios are indicated by the following statistics. Only 3 of 16 adults said that the vertical line was longer for the 1.0 or smaller ratios in the 80-deg threedimensional viewing condition (binomial probability, $\mathrm{p}<.05$ ). The 60 -deg adult three-dimensional viewing condition and each of the 40-deg conditions did not show significant regression of the apparent equal ratios from the equal three-dimensional ratios by this test $(p>.05)$. Similar analyses indicated that all conditions were significantly different from the equal picture-plane ratios $(\mathrm{p}<.05)$.

As a group, the children were less consistent in their length judgments, which was indicated by a greater tendency to shift judgments in the series more than once. For example, a child might judge the 4.0, $3.0,2.0$, and 1.75 ratios as vertical-longer, and the 1.5 ratio as vertical-shorter, and then return to a vertical-longer judgment for the 1.25 ratio. Eight children responded with a total of 13 reversals and one adult responded with 2 reversals.

\section{DISCUSSION}

The results will be considered from two points of view. First we will examine the general compromise in length judgments between the three-dimensional ratios and the picture-plane ratios. Then we will compare the two age groups and address the developmental questions that were raised in the introduction.

\section{Perceptual Constancy and "Compromise"}

Adult abilities to achieve perceptual constancy with pictorial depth and slant information have been demonstrated in a variety of experiments. One recent contribution to this literature was Lappin and Preble's (1975) demonstration that shape constancy for irregular figures photographed at various slants from the picture-plane could be maintained when they were presented on a pictorial background surface which contained a variety of cues for depth and slant. Our study took a different approach to the constancy problem by presenting a single pictured object (a box) whose three-dimensional orientation could be specified by an assumption (conscious or unconscious) that the contours belonged to a three-dimensional shape with rectangular surfaces. Such shape perspective information for depth and slant is a special case of the broader range of pictorial variables which are informative because of their typically invariant relationships to depth and orientation in the threedimensional world (Gibson, 1966).

Shape perspective produced length judgments from our pictures which were closer to the threedimensional values than the picture-plane values, but constancy was not complete. What caused the regression of length judgments toward the pictureplane lengths, and how does this result relate to those of other perceptual constancy experiments?

Thouless (1972) has noted that the judged shape of an object is typically a "compromise" between the three-dimensional shape and the projective shape, and this "compromise" shifts toward the projective shape as depth cues are removed. With random shapes such as those used by Lappin and Preble, the complete removal of the picture background would probably lead to a projective shape judgment. However, the box shape employed in the present study carried perspective information for its own orientation, and constancy was partially maintained with the slides, without the support of other pictorial depth cues.

The incomplete constancy found in our experiment may be related to the results from previous studies of slant responses to two-dimensional outlines (Attneave \& Frost, 1969; Olson, 1974). These experiments found that even under optimal viewing conditions, slant judgments tend to regress toward the picture plane from the perspective implied slant. The regression of length judgments toward picture plane values in the present study could have been caused by misperceived slant of the box surface, and it could also be related to the subject's perception of the box dimensions. We noticed that the box dimensions 
seemed different in the different orientations and the reader may verify our observations in Figure 1 . The box in the lower right corner of the figure could almost be mistaken for a cube, although the top surface of the three-dimensional box was twice as long as the front face. Likewise, the lower-left box seems to have a width-to-length ratio closer to $1: 2$ rather than the actual 1:4 ratio. We verified our observations by presenting the boxes in Figure 1 in different random orders to 28 adult subjects under viewing conditions approximating those in the present experiment. The subjects made ratio judgments of the width of the front face and the length of the top face. The resulting judgments were averaged and the ratios transformed to correspond to the values in Figure 2. At the 40-deg box angle, the perspective distortion ratio averaged 1.0 , thus indicating a mean response ratio that was very close to the actual threedimensional ratio. At $60 \mathrm{deg}$, the distortion ratio was 1.29 , and at $80 \mathrm{deg}$ it was 1.66 . Thus, the box dimension distortion ratios were quite close to the cross-arm distortion ratios in the main experiment (see Figure 2), and the regressions in length judgments toward the picture-plane may be related to the perceived box angle, the perceived box length, or both.

\section{Developmental and Theoretical Issues}

Length judgments in the slide-viewing condition were remarkably similar between the two age groups. Thus, the children, although somewhat more variable considering the frequency of reversals, were not significantly different from the adults in their use of shape perspective information. Several children between 3 and 4 years of age were tested to reveal possible earlier developmental differences, but the results were quite variable, and it was difficult to maintain the children's attention to the task. Two children ( 3 years, 4 months and 3 years, 7 months of age) completed the whole trial series. The mean transition points in their longer-shorter judgments were similar to those for the older subjects in the main experiment.

The children in our sample had considerable contact with shape perspective invariances in their natural environment as well as in pictorial representations on television and in picture books. Empiricist theories regarding the origins of sensitivity to perspective information emphasize the role of experience (Ames, 1951; Brunswik, 1956). Thus, learning to perceive slant and depth from shape perspective is a matter of remembering (consciously or unconsciously) the previous associations between particular perspective views and particular orientations which were somehow specified by other information. This view would seem to place a fairly heavy burden on the developing child to "remember" each of his previous associations with the multitude of particular perspectives for particular shapes and particular slants. It would also seem to predict a gradual development of sensitivity to shape perspective, a prediction the present study does not support, at least past 4 years of age.

Another view of the basis for slant and depth responses to shape perspective is that the observer seeks the simplest possible description of visual information. If a pictured collection of contours may be more simply described in three dimensions than in the two-dimensional picture plane, it will tend to be seen in depth (Hochberg \& Brooks, 1960). Moreover, with respect to shape perspective, the object's perceived orientation is related to the threedimensional orientation which allows for its simplest description (Attneave \& Frost, 1969).

The early Gestalt approach to organizational simplicity emphasized' its basis in the innate characteristics of the nervous system (Koffka, 1935). However, the simplicity hypothesis need not exclude experience with shape perspective invariances from playing some role in the development of pictorial depth and slant responses. Instead of learning a series of associations with particular perspective views and particular slants, the developing child may learn abstract rules regarding the three-dimensional regularities of certain objects, and perhaps some general operations for the regularization of two-dimensional projections in a three-dimensional mental analog space. This would allow the generation of slant and depth from a perspective view without calling on previous associations with that particular perspective. At the present time, we do not have a precise model of how the nervous system might accomplish this sort of operation, although Attneave (1972) has proposed a general analog model in support of the simplicity theory.

The results from children in the present study do not provide for a definite choice between the empiricist-associationist and generative-simplicity theories. However, our failure to find a developmental difference suggests that the ability to respond to shape perspective does not require prolonged experience with perspective-three-dimensional invariances.

\section{REFERENCE NOTES}

1. Yonas, A. The pickup of shadow information in pictures by infants and children. Paper presented at the Conference on Picture Perception. University of Minnesota, July 1975.

2. Olson, R. K. Unpublished studies on childrens' response to shape perspective. University of Colorado, 1973.

\section{REFERENCES}

Ames. A. Visual perception and the rotating trapezoidal window. Psychological Monographs, 1951, No. 324.

Atrneave, F., \& Frost, R. The determination of perceived tridimensional orientation by minimum criteria. Perception \& Psychophysics, 1969, 6, 391-3\%. 
Attweave, F. Representation of physical space. In A. W. Melton \& E. Martin (Eds.) Coding processes in human memory. New York: Winston, 1972.

BowER, T. G. R. Stimulus variables determining space perception in infants. Science, 1965, 149, 88-89.

Brunswick. E. Perception and the representative design of psychological experiments (2nd ed.). Berkeley: University of California Press, 1956.

DAy, R. H. Perceptual processes in early infancy. Australian Psychologist. 1974, 9. 15-34.

GiB SON, J. J. The senses considered as perceptual systems. Boston: Houghton Mifflin, 1966.

HochBerG. J. E., \& Brooks, V. The psychophysics of form: Reversible-perspective drawings of spatial objects. American Journal of Psychology, 1960, 73, 337.354.

KoffKa, K. Principles of Gestalt psychology. New York: HarcourtBrace, 1935.
Lappin, J. S.. \& Preble, L. D. A demonstration of shape constancy. Perception \& Psychophysics, 1975, 17, 439-444.

Olson, R. K. Slant judgments from static and rotating trapezoids correspond to rules of perspective geometry. Perception \& Psychophysics, 1974, 15, 509-516.

Olson, R. K. Children's sensitivity to pictorial depth information. Perception \& Psychophysics, 1975, 17', 59-64.

Orson, R. K., \& Boswell. S. L. Pictorial depth sensitivity in twoyear-old children. Child Development, 1976, in press.

Thouless, R. H. Perceptual constancy or perceptual compromise. Australian Journal of Psychology. 1972, 24, 133-140.

(Received for publication February 18, 1976; revision accepted June 21. 1976.) 